\title{
WELL DIFFERENTIATED TUBULAR ADENOCARCINOMA INVASIVE COLON PRESENTING AS A DERMATOMYOSITIS: CASE PRESENTATION AND REVIEW OF LITERATURE
}

Raylane Shellyda de Almeida Anate ${ }^{1, *}$, Larissa Fernandes Antunes² ${ }^{2}$ Themis Mizerkowski Torres ${ }^{1}$

1.Faculdade IPEMED de Ciências Médicas, São Paulo (SP), Brazil. Universidade de Taubaté, Taubaté (SP), Brazil.

*Corresponding author: raymed87@gmail.com

\section{BACKGROUND}

Inflammatory myopathies, which include dermatomyositis (DM) and polymyositis (PM), are chronic systemic diseases associated with high morbidity and functional disability, which mainly affect skeletal striated muscles. It is often the first sign of an underlying neoplasm, musculoskeletal complaints of acute onset, early and intense skin manifestations as well as the presence of cutaneous necrosis are often the first symptoms, being more common lesions in photoexposed areas, proximal muscle weakness, changes in respiratory muscles and dysphagia. Current treatment is based on corticosteroid therapy and the use of immunosuppressants, but a considerable portion of patients are refractory to traditional therapy. This has led to the attempted use of immunobiological drugs. To date, anti-CD20 therapy seems to be the most promising. Rehabilitation and physiotherapy measures are critical elements to be included in each stage of treatment. Physical exercise in conjunction with pharmacological treatment is safe and effective in improving patient clinical outcomes.

\section{CASE REPORT}

A.A.A., 68 years old, male, born in Andirá (PR), resident of Taubaté (SP), a civil engineer, presented in April 2015 picture of erythema and facial edema, hypothetically associated with contact with antigenic agents due to the function he performed in his employment, was treated with corticosteroids that resulted in moderate improvement of the condition. Times ahead evolved with the presence of heliotrope, Gottron papules, paronickel in all fingernails (Fig. 1), shawl sign, ulcerated lesions on the skin in the cervical region (Fig. 2), thoracic, inguinal and sacral region, intense cephalic itching, and, weeks later, with myopathy. Muscle symptoms were myalgia, important weakness of the proximal muscles of the coxofemoral joints and humerus scapular, alteration in phonation mechanics and progressive high dysphagia. It was then investigated clinically and laboratory and diagnosed as dermatomyositis. Treatment with azathioprine and methodrex concomitantly associated with oral corticosteroids was then initiated, but the patient did not meet treatment expectations and continued to evolve with myopathy and intense weight loss. Further research was needed to find out why the response was inadequate. For screening, a stool occult blood test was requested (positive), followed by colonoscopy that showed a colon adenocarcinoma. Surgical removal was indicated to treat the tumor, which was impossible to be made at the patient1s first hospitalization due to the presence of febrile neutropenia. After improvement of the condition and slight weight gain, the surgery was performed. At this time, the patient was still with the aforementioned medications. During hospitalization, the patient progressed poorly, and a new reapproach was needed after fistula identification. After the second approach, the patient continued to evolve poorly, presenting an acute pulmonary edema and being referred to the ICU. During the period in the intensive care unit, the patient presented low gastrointestinal bleeding and respiratory failure, requiring IOT. He progresses well, being discharged to the infirmary and concomitantly to domiciled. After discharge, the patient progressed well, with no signs of active rheumatologic disease and gradual improvement of skin lesions. The medications azathioprine and methotrexate were withdrawn, as well as corticosteroids gradually, but when the corticosteroid was at a dose of $5 \mathrm{mg} /$ day, there was a recurrence of the disease, and it was necessary to resume the dose of $40 \mathrm{mg} /$ day and start hydroxychloroquine. During the rheumatologic disease activation, there was a new investigation of oncological diseases that was negative. Due to the improvement of the patient's condition, the gradual removal of the corticosteroid and total removal of hydroxychloroquine was resumed. In June 2018, the patient was already without any medication and remained without any sign of rheumatologic disease to date. For follow-up, the patient performs laboratory tests every two months to analyze disease activity, and clinical evaluation. 


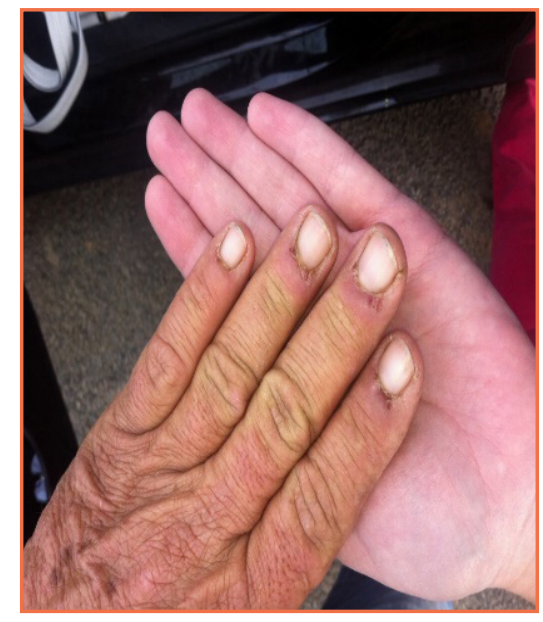

Figure 1. Presence of heliotrope, Gottron papules, paronickel in all fingernails.

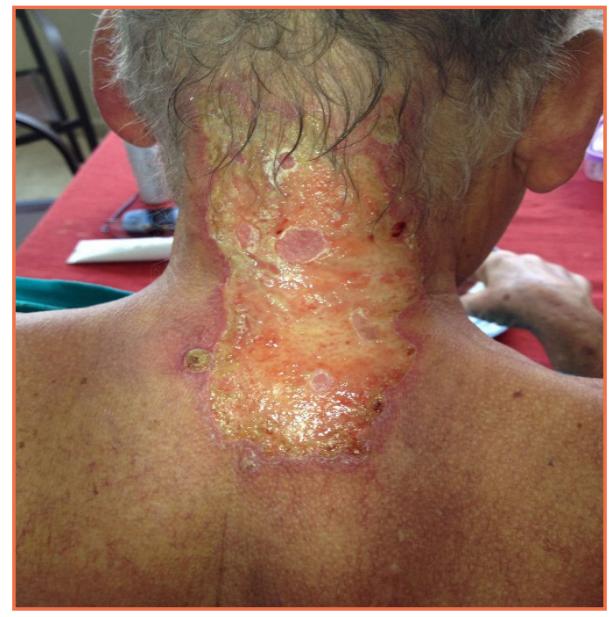

Figure 2. Shawl sign, ulcerated lesions on the skin in the cervical region.

\section{CONCLUSION}

Dermatomyositis is an idiopathic connective tissue disease characterized by specific cutaneous findings and inflammatory lesions in muscle biopsy. An association between dermatomyositis and malignancy, including breast, ovarian, lung and colon cancer was recognized many years ago, with an incidence of malignancy in approximately $20 \%$ of cases. Finally, it is essential to perform a systemic survey of malignancy in patients with DM, since there is a high incidence of cancer. Malignancy therapy has to precede DM therapy, as DM will possibly be cured by a successful treatment of malignancy. 